\title{
COST Action FA1401 "European network on the factors affecting the gastro-intestinal microbial balance and the impact on the health status of pigs (PiGutNet)"
}

\author{
P. Trevisi' ${ }^{1,9}$, S. Botti ${ }^{2}$, C. Lauridsen 3 , J.F. Pérez ${ }^{4}$, D. Papadopoulos ${ }^{5}$, M. Roselli6, \\ J. Levic ${ }^{7}$ and J. Zentek ${ }^{8}$ \\ 1 University of Bologna, Department of Agricultural and Food Sciences (DISTAL) \\ Viale G. Fanin 46, 40127 Bologna, Italy \\ ${ }^{2}$ Parco Tecnologico Padano, Via Einstein, Loc. Cascina Codazza, 26900, Lodi, Italy \\ ${ }^{3}$ Aarhus University, Department of Animal Science, Blichers Alle 20,8830 Tjele, Denmark \\ ${ }^{4}$ Universitat Autònoma de Barcelona, Departament of Animal and Food Sciences, Animal Nutrition and Welfare Service (SNiBA). \\ 08193 Bellaterra, Spain \\ ${ }^{5}$ Aristotle University of Thessaloniki, Faculty of Health Science, School of Veterinary Medicine \\ 54124 Thessaloniki, Greece \\ ${ }^{6}$ Research Center on Food and Nutrition (CRA-NUT), Agricultural Research Council \\ Via Ardeatina 54600178 Rome, Italy \\ ${ }^{7}$ University of Novi Sad, Institute of Food Technology, Bul. cara Lazara 1, 21000 Novi Sad, Serbia \\ ${ }^{8}$ Freie Universität Berlin, Department of Veterinary Medicine, Institute of Animal Nutrition \\ Königin-Luise-Str. 49, 14195 Berlin, Germany
}

KEY WORDS: pigs, microbiome, gut health, antibiotic resistance, animal health and welfare
ABSTRACT. The "hoped for" reduction in the use of antibiotics in pig by EU producers has not been materialized as they are still being widely used for the control of enteric infectious diseases. This practice can spread antibiotic resistance in the farm environment and poses a threat to consumer health. Whilst it is widely recognized that a diversified gastro-intestinal tract (GIT) microbiota is essential for optimal health and performance, the underlying factors favoring the development and maintenance of a balanced intestinal microbiota are not fully understood. PiGutNet will establish the first European network focused on this topic, joining specialists in all research areas. It will define both environmental and host genetic factors affecting the GIT microbiota and the complex interactions between microbiota and gut maturation, to maintain a healthy gut throughout life. The network will coordinate databases and unravel innovative tools to define the status of intestinal eubiosis in pigs. The most important outcomes will be genome/metabolome-wide association studies and the provision of a road map to increase pig resistance against GIT infections. This will have an important translational potential, being the foundation for European companies to develop strategies in the areas of feed additives and animal husbandry, resulting in improved animal health and welfare, consumer protection and competitive advantage for the European agriculture.
${ }^{9}$ Corresponding author: e-mail: paolo.trevisi@unibo.it 


\section{Brief overview of the COST Action program}

COST Actions are flexible, fast, effective, competitive and efficient networking instruments for scientists, engineers and scholars to cooperate and coordinate nationally funded research activities. COST Actions allow European researchers to jointly develop their own ideas in any science and technology field. COST has been contributing - since its creation in 1971 - to closing the gap between science, policy makers and society throughout Europe and beyond. As a precursor of advanced multidisciplinary research. The COST Action framework includes 9 scientific domains, specific for different topics. Moreover, it is possible to apply for a so called Trans- Domain Action in which different domains should be included to solve transversal issues (http://www.cost.eu/COST_Actions). The Food and Agriculture (FA) domain covers all aspects of research in the field of agricultural and food sciences in its widest sense. The primary aim of the Domain is to encourage networking of research in any field linked to these activities as well as the related demands and needs. Several networks were created in the FA domain in the last decades to increases the knowledge in specific topic of high relevance for the agricultural science. During 2014 COST Action FA1401 "European network on the factors affecting the gastro-intestinal microbial balance and the impact on the health status of pigs (PiGutNet)" was established in this Domain, and was finally approved in January 2015. Our PiGutNet started its activities after with the kick-off meeting in Brussels, October 2014. The COST Action budget is fully dedicated for the networking activities, the dissemination of the knowledge generated from the network and to promote the mobility of the young researchers between the institutions involve in the Action. Conversely, no research activities are supported by the COST Action budget.

\section{Background of PiGutNet network}

The European Union was among the first in the world to introduce strong limitations in the use of antibiotics in livestock breeding. Since 2006, the use of antibiotic as in-feed growth promoter has been banned. Furthermore, several restrictions in the use of antibiotics for therapy have been progressively introduced in the last decade. Similar to guidelines for the use of antibiotic drugs in human medicine, the use of these molecules should be motivated and carefully supervised also in pig. The reasons of this policy are founded in the goal to contain the spread of the antibiotic resistance that is the main threat to reduce the effectiveness of the antibiotic therapy in livestock, increasing the risk of zoonosis occurrences in humans. The extent to which antibiotic resistance in animal agriculture contributes to human infections is not known.

This policy strongly impacts the livestock productive systems, in special ways, for species reared in intensive conditions, like pigs. In fact, gastrointestinal tract disorders remain major problems, and the use of therapeutic intervention is still being widely used in the control of infectious and other enteric diseases. On the other hand, antibiotic resistance is recognized as a world threat, and for this reason, FAO's Animal Production and Health Division (AGA) supports measures to minimize and contain antimicrobial resistance.

The European Union supported research to increase animal health and individuate alternatives to in feed antibiotic (for example "Healthypigut" QLK5-CT-2000-00522, "Feed for Pig Health" FOOD-CT-2004-506144, "INTERPLAY" Agreement No. 227549, "QUALITYLOWINPUTFOOD"

FP6-FOOD-CT-2003-506358). Thanks to these projects, new knowledge was acquired on the gut physiology, immunology and microbiology of pigs. Many scientific papers were published and a large number of data are available.

It was evident for example that a proper balance in the gut microbiome is important to reduce the need for therapy in the breeding of young pigs, and also that several management factors affect the abundance of some dominant bacterial species in the porcine GIT, including manipulation very early in pig's life and antibiotic supplementation of the mother. Thus, improper management procedures can contribute to a sub-inflammatory condition and bacterial dysbiosis in the gut.

However these studies also often highlighted the limit of traditional analytical tools and showed the opportunities created by new molecular highthroughput technologies, and thereafter the need for new standardization. Moreover, within some research groups it is also intensively discussed the difficulty to integrate all the new potential knowledge related to the extraordinary quantity of genomic data becoming available for domestic animal species.

This critical aspect was also evident in several international meetings, where a large number of the promoters of PiGutNet participated. On these occasions clearly emerged the need for an integrative network among scientists of different disciplines to focus on a possible common starting point on the 
problems related to porcine gut health. This was the first seeding of the idea to explore the COST Action as a suitable instrument to work together. This structure created opportunities to gather scientists and industries having the pig health as their main interest, from various disciplines and from a large number of research fields to establish a multidisciplinary network with the necessary critical mass to translate the acquired knowledge into practical applications.

Afterwards, contacts were established with all major research centers in Europe working in pig science, in order to create an international framework that will benefit European scientists.

The objectives were defined according to the needs of the sector - in the new scenario derived by the sequencing of the pig genome - that should be pushed in a post-genomic context in order to fully exploit this resource.

The pre-proposal was planned just after the meetings occurred at the $20^{\text {th }}$ National Congress of the Italian Scientific Association for Animal Production (ASPA) (11-13 June 2013) and was immediately supported by research groups working in 30 institutions from 21 COST Action countries and several end-users. Actually, 46 institutions, 10 companies, 5 breeding associations and the European Federation for Animal Science (EAAP) of 22 EU countries (Austria, Belgium, Croatia, Czech Republic, Denmark, France, Germany, Greece, Ireland, Italy, Netherlands, Norway, Poland, Portugal, Romania, Serbia, Slovakia, Spain, Sweden, United Kingdom, Switzerland, Albania) and 3 extra-UE counties (Australia, Canada and China) are involved in the COST Action.

\section{Objectives and problem to solve}

This Action aims to advance the knowledge across all the areas related to gut microbial variation, epigenetic modifications, and expression profiling for the enhancement and advancement in the sustainability of the livestock production. The main objective of PiGutNet is to increase the knowledge about the effect/interaction of environmental and genetic factors on the composition of the microbiota in the gastrointestinal tract of pigs and to improve the risk management associated with antibiotic resistance in pig production. The idea of "balanced" or "eubiotic" microbial gut colonization is the overall target and needs conceptual definition and methodological backup. Moreover, accordingly to the aims of the COST Action program, PiGutNet network has the key objective to favor the mobility of the young researches ( $\mathrm{PhD}$ and Post-Doc) between the facilities of the research groups involved in the network to speed up their career with attention to balance the opportunities for all the people involved in the Action (gender and country balance).

Concerning this last point, Training Schools and Short Term Scientific Mission are sponsored by the Action. While, for the scientific point of view, in order to disentangle all the factors involved in the gut microbial balance the four gaps listed below need to be taken into account by the network (Figure 1):

4 GAPS TO CONSIDER:

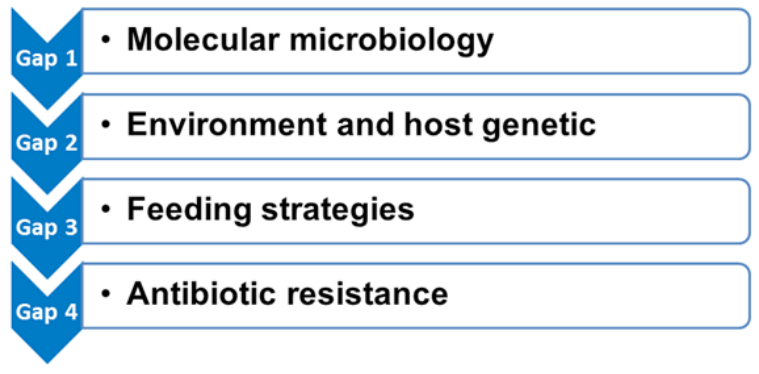

Figure 1. Research areas to consider in the study of the gastrointestinal microbial balance

\section{Molecular microbiology}

Thanks to next generation sequencing (NGS) techniques, in a few years the full genomes of a wide number of bacterial strains have been sequenced. These data will be highly relevant for bacterial characterization and will increase the knowledge on diversity, taxonomy and, where relevant on the pathogenesis of a particular bacterial infection. Moreover, these databases will provide the opportunity to study changes in bacterial metabolism under different gut conditions (enterotype approach). Using this approach PiGutNet will deepen the interactions between the microbiota and all the factors modifying its composition and function. Numerous protocols have been published based on different techniques/instruments, and in order to facilitate data sharing a standardized approach will be promoted. Metabolomic approach will be integrated, as compositional data maynot always reflect the situation of the gut microbiota.

\section{Environment, host genetics and epigenetic approach}

The gut homeostasis is a delicate equilibrium. To improve the risk management linked with gut dysbiosis, it will be necessary to highlight all the factors involved in the maintenance of an optimal 
microbial balance or, conversely, the factors increasing the destabilization of the gut homeostasis. For environmental factors there is a need to expand knowledge, whereas to date there is no consensus on the relevance of host genetic effects. Thus the network reviews the present knowledge and provides new models to integrate the host genomics into the gut phenotypes studies. Furthermore, it can be suspected that some environmental effects can be replicated between generations. Hence it would be relevant to focus on the sow-piglet relationship. Based on recent findings, it has been suggested that intrauterine growth retardation (IUGR) could play a central role in the proper development of gut function and gut tissues maturation.

\section{Feeding strategy to manage the risk of dysbiosis}

The pressure of the EU ban on the use of infeed antibiotics stimulated studies on the development of feeding strategies to control gut microbiota, mostly focusing on other functional feed ingredients and additives predicted as having their main action through pro- or antibacterial activity. Less interest, however has been given to feeding strategies that could help the host to develop a more stable and favourable gut environment from birth and thereby exploit the natural ability of piglets to develop their gut environment, reducing the risks of dysbiosis in suboptimal rearing conditions. This gap will be filled by focusing more on piglet intervention measures immediately from birth, considering the integrated system piglet-sow-weaning-post-weaning, instead of concentrating only upon feeding during the post-weaning period. In particular this could be obtained in PiGutNet: 1) collecting and discussing the new evidence on the host ability to establish an interplay with the developing microbiota, including all data about pig gut transcriptomic and proteomic; 2) suggesting new dietary intervention techniques to exploit this new knowledge; 3 ) considering reliable strategies to induce precocious GIT maturation before weaning, to achieve the characteristics of the post-weaning condition and 4) incorporating other promising feeding practices present in different geographic regions of the EU.

\section{Antibiotic resistance}

This research area task aims to develop research targeted to assess: the development of antibiotic resistance (resistome) in pigs, the emergence of multidrug-resistant Enterobacteriaceae and possible dietary and management strategies to reduce the spreading of antibiotic resistance genes among bacteria at farm level. PiGutNet will target the data collection and generation to better understand this complex picture and contribute to the identification of the main factors characterizing farms using high amounts of antibiotics from those not using antibiotics. This analysis could help providing more awareness about the prudent use of antibiotics in veterinary medicine and the importance of the development antibiotic free farms in the future.

\section{Scientific Organization}

The 4-years PiGutNet program will be carried out within 5 working groups (WGs) identified according to the 4 main research areas above described, while one WG will be dedicated to the dissemination (Figure 2).

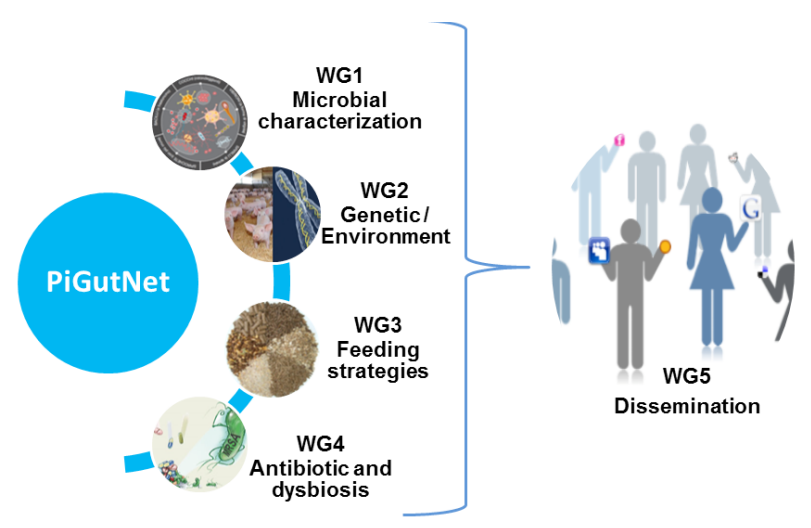

Figure 2. Working groups organization

WG1. Functional and genetic characterization of microbial communities in the gastrointestinal tract of pigs. The ability to fully characterize the intestinal ecosystem is the cornerstone to understand the effectiveness of practical solutions to manage the intestinal homeostasis and to improve the health status and disease resistance of the animals. The main objectives of WG1 are to compare different procedures to characterize the microbiome under the new light of the metagenomics approach in the GIT of pigs to define its composition and functionality at different production phases under experimental conditions (optimized housing and feeding conditions) and to define the status of eubiosis and dysbiosis. 
WG2. Genetic and environmental factors to understand dysbiosis including their interaction (epigenetics). Dysbiosis is considered a contributing cause of GIT diseases and must be discussed as a function of genetics and husbandry factors. Therefore, in this WG, geneticists, microbiologists, physiologists, nutritionists and immunologists will work together to improve the knowledge on the genetic potential of the typically used pig breeds in European production systems, to establish strategies to increase the resistance against pathogens, with special emphasis on the immune response and gut maturation and to define the most important phenotypes related to the gut microbiota and the best ways to collect/register them. Moreover, the partners will define the environmental factors (mother imprinting, diet, housing, etc.) that cause dysbiosis and their interaction with the physiological and genetic make-up of pigs' (epigenetics) parameters.

WG3. Feeding strategy to maintain/restore the gut homeostasis. Diet composition and feeding strategy both play important roles in the development and establishment of the gut microbiota in terms of function and composition. They provide the opportunity to manage the risk associated with inheriting a genetic predisposition to a particular infection (e.g. adhesion determinant) without the use of antibiotics. There is a lack of understanding of the mechanisms underlying these effects, and this aspect remains a pre-requisite to establish if possible benefits are to be developed in a practical "on-farm" solution.

WG4. Antibiotics as a factor of dysbiosis and spread of antibiotic resistance genes. This WG is especially dedicated to the causes of the development of antibiotic resistance (resistome) in pigs and in particular the emergence of multidrug-resistant Enterobacteriaceae and Staphylococcaceae. A second aim is to involve businesses in practice and to investigate the association among farming systems, genetics, feeding, veterinary care and the use of chemotherapeutic agents. Here the influence of the genetic make-up and the different levels of biosecurity in swine farms will discussed with regards to the increase in antibiotic resistance among pathogenic and bacteria of the commensal flora.
Moreover an attempt to assess the impact of the misuse of antibiotics in bacterial variability as well as in health and productivity of swine production systems, will be made.

WG5. Knowledge and management exchange (KME). This is a key WG devoted to improve the exchange of experiences and knowledge, across the WG1 to WG4, and to manage the dissemination towards external stakeholders in order to spread the information generated from the network.

\section{Expected impact}

We hope to advance the European research strategy on animal health and welfare in domestic animals by the activities, to create a sustainable network of experts in pig nutrition and to foster transdisciplinary discussions, research and future projects in an important domain. PiGutNet will stimulate and support the European Union's needs and societal demands and by this increase the competitiveness in the agricultural sector.

\section{Contact and update}

The PiGutNet network is open to accept new partners that aim to share information to progress in the field of the gut microbial balance of pigs. In order to have more information on the network (partner involved, Management Committee composition, scientific objectives, activities, etc.), please, visit our webpage http://www.cost.eu/COST_Actions/ fa/Actions/FA1401 and www.pigutnet.eu. Moreover, you can contact the Chair and the Vice-Chair of the PiGutNet network, Prof. Paolo Trevisi from the University of Bologna and Prof. Jürgen Zentek from the Freie Universität Berlin.

\section{Acknowledgement}

The authors would like to acknowledge networking support by the COST Action FA1401 